\title{
Specific interaction of methionine adenosyltransferase with free radicals
}

\author{
M.A. Avila, F.J. Corrales, F. Ruiz, E. Sánchez-Góngora, J. Mingorance, M.V. Carretero and \\ J.M. Mato* \\ Departamento de Medicina Interna, Facultad de Medicina, Universidad de Navarra, \\ 31080 Pamplona, Spain
}

Received 10 July 1997

Revised 24 November 1997

Accepted 3 December 1997

\begin{abstract}
Although free radicals have been traditionally implicated in cell injury, and associated to pathophysiological processes, recent data implicate them in cell signaling events. Free radicals are naturally occurring oxygen-, nitrogen- and sulfur-derived species with an unpaired electron, such as superoxide, hydroxyl radical or nitric oxide. In order to assess the role of free radicals in cell signaling, we have studied the modulator effect of oxygen and nitrogen active species on liver methionine adenosyltransferase (MAT), a key metabolic enzyme. The presence of 10 cysteine residues per subunit, makes liver MAT a sensitive target for oxidation/nitrosylation. Here we show that purified MAT from rat liver is nitrosylated and oxidized in vitro. Incubation with $\mathrm{H}_{2} \mathrm{O}_{2}$ or the $\mathrm{NO}$ donor S-nitrosylated GSH (GSNO), diminish MAT activity in a doseand time-dependent manner. Furthermore, the inactivation derived from both oxidation and nitrosylation, was reverted by GSH. MAT inactivation originates on the specific and covalent modification of the sulphydryl group of cysteine residue 121 . We also studied how free radicals modulate MAT activity in vivo. It was previously shown that MAT activity is strongly dependent on cellular GSH levels. Generation of oxygen and nitrogen active species in rats by injection of LPS, induced a decrease of liver MAT activity. This effect might derive from nitrosylation and/or oxidation of the enzyme. Modulation of liver MAT by NO is further supported by the inactivation of this enzyme observed in experimental models in which $\mathrm{NO}$ is produced; such as the administration of $\mathrm{NO}$ donors to rats and in hepatocytes cultured in hypoxia, a condition that induces the expression of the inducible nitric oxide synthase (iNOS). Oxidation also controls liver MAT activity in a cell environment as shown in $\mathrm{CHO}$ cells stably transfected with rat liver MAT cDNA upon addition of $\mathrm{H}_{2} \mathrm{O}_{2}$ to the culture medium. This effect depends upon the generation of the hydroxyl radical. On the basis of the metabolic implications of liver MAT, together with the structural features accounting for the sensitivity of this enzyme to active oxygen and nitrogen species, we propose that modulation of MAT by these agents could be a mechanism to regulate the consumption of ATP in the liver, and thus preserve cellular viability under different stress conditions.
\end{abstract}

\section{Introduction}

Free radicals have been traditionally implicated in cell injury; however, a physiological role for these agents is currently being unveiled. Apart from the participation of reactive oxygen and nitrogen species in processes such as the immune response [10] and the regulation of the vascular tone [7], free radicals seem to play an important role in cellular signaling [8]. This new function is supported by

\footnotetext{
${ }^{*}$ To whom correspondence should be addressed: J.M. Mato, Departamento de Medicina Interna, Facultad de Medicina, Universidad de Navarra, 31080 Pamplona, Spain.
}

0951-6433/98/\$8.00 @ 1998 - IOS Press. All rights reserved 
several lines of evidence. First is the growing list of cellular events that free radicals can modulate [8]; among which we find most of the well-characterized cell signaling cascades. Stronger support for a role of free radical as cellular messengers comes from the observation that growth factors, such as platelet-derived growth factor (PDGF), need to produce reactive oxygen species in order to mediate their cellular effects [12].

The identification of intracellular targets for reactive oxygen and nitrogen species is critical in the elucidation of how these agents propagate their signals. Moreover, this knowledge is relevant to the pressing question of how free radicals achieve specificity in their effects.

We have now identified methionine adenosyltransferase (MAT) as a target for reactive oxygen and nitrogen species. This enzyme catalyzes the first step of methionine metabolism producing, in the presence of ATP, the major biological methylating agent: S-adenosylmethionine (AdoMet). In mammals, two different genes encode for MAT, one being expressed only in liver while the other is expressed in extrahepatic tissues and fetal liver [9]. Liver MAT can be found as two oligomeric forms, a dimer (MATIII) and a tetramer (MATI) of the same subunit, while extrahepatic MAT exists only as a homotetramer (MATII). The deduced aminoacid sequence of the subunit of the liver enzyme shows the presence of 10 cysteine residues, which are conserved in rodents and humans [9]. This makes MAT a potential target for regulation through thiol group modification [9]. MAT activity is specially high in liver tissue, where the majority of methionine is metabolized. Impairment of methionine metabolism has been demonstrated in different liver disorders and experimental models which compromise hepatic function, such as liver alcoholic and viral cirrhosis, chronic ethanol consumption, sepsis and hypoxia [9]. An event common to most of these pathological situations is the generation of oxygen and/or nitrogen free radicals. Here we report on our current studies about the interaction of MAT with nitrogen and oxygen reactive species and discuss the physiopathological implications of MAT regulation by such agents.

\section{MAT activity during oxidative and nitrosative stress in vivo}

Early evidence for the implication of free radicals as mediators of MAT inactivation in liver disease was provided by the study of MAT activity in experimental models of liver injury, such as $\mathrm{CCl}_{4}$ and ethanol chronic intoxication (reviewed in [9]). The generation of free radicals is a common event in these two models of liver injury, and is thought to play a key role in the induction of liver damage by both agents. Moreover, it has been demonstrated that MAT activity depends on the intracellular concentration of reduced glutathione (GSH). When GSH synthesis was inhibited with buthionine sulfoximine (BSO, an inhibitor of GSH synthesis by inactivation of $\gamma$-glutamylcysteine synthetase), MAT activity was decreased. MAT inactivation could be prevented by the administration of GSH-ethyl ester (a permeable derivative of GSH) together with BSO [5].

More recently, we have shown that MAT can be inactivated in the absence of GSH depletion. In a model of septic shock, in which bacterial lipopolysaccharide (LPS) is injected into rats, liver MAT activity was significantly diminished, while GSH levels remained constant [1]. It is known that LPS triggers the generation of activated oxygen and nitrogen species in liver. Indeed, we observed a marked induction of the superoxide-metabolizing enzyme, superoxide dismutase (MnSOD) and the NO-producing enzyme, inducible NO synthase (iNOS), upon injection of LPS. In order to explore the role of reactive nitrogen species in MAT inhibition we treated rats with the NO donor 3-morpholinosydnonimine (SIN-1). The administration of this molecule resulted as well in a substantial loss of liver MAT activity, thus demonstrating that MAT inactivation in sepsis might be mediated 
by NO. However, reactive oxygen species, as we previously mentioned, are also generated during sepsis. The final outcome in terms of in vivo MAT inactivation, could be the result of the interaction between nitrogen and oxygen species among themselves and with MAT sulphydryl groups [2].

In order to assess individually the role of oxygen and nitrogen activated species in MAT inhibition in vivo, we have used different experimental models. The action of reactive oxygen species was tested in $\mathrm{CHO}$ cells which had been stably transfected with rat liver MAT cDNA [11]. In this model MAT was inactivated by the addition of $\mathrm{H}_{2} \mathrm{O}_{2}$ to the culture medium. This effect was dependent upon the generation of the hydroxyl radical, since MAT inactivation by $\mathrm{H}_{2} \mathrm{O}_{2}$ could be prevented by the iron chelator desferoxamine. Interestingly wild type $\mathrm{CHO}$ cells, which only express the extrahepatic isoenzyme of MAT (MATII) were resistant to $\mathrm{H}_{2} \mathrm{O}_{2}$-mediated inhibition. Noteworthy, only 5 out of the 10 cysteine residues present in the hepatic form are conserved in MATII.

The interaction of MAT with NO in the absence of reactive oxygen species was further characterized in another experimental model. Working with cultured rat hepatocytes, we have observed the induction of iNOS upon expossure to low oxygen levels $\left(3 \% \mathrm{O}_{2}\right)$. In this model of cellular stress oxygen free radicals are not produced [3], however, MAT results inactivated after $6 \mathrm{~h}$ of incubation in hypoxia. A role for NO in MAT inactivation under hypoxia was further demonstrated by the use of the iNOS specific inhibitor $\mathrm{N}^{\mathrm{G}}$-monomethyl-L-arginine methyl ester (L-NAME). When this agent was added to the culture medium during hypoxia, MAT inactivation was prevented.

Taken together our results indicate that MAT is a sensitive target for free radicals in vivo. Furthermore, MAT inactivation seems to be a common response to a diverse set of pathological and experimental situations, in which free radicals are generated. On the other hand, MAT sensitivity to these agents is apparently restricted to the hepatic isoenzyme, given the lack of inhibition observed in wild type $\mathrm{CHO}$ cells treated with $\mathrm{H}_{2} \mathrm{O}_{2}$.

\section{MAT interaction in vitro with reactive oxygen and nitrogen species}

In order to gain more insight into how oxygen and nitrogen activated species interact with MAT, we carried out in vitro studies in which rat liver purified MAT was challenged with these agents.

The incubation of MATI and MATIII with the NO donors SIN-1 [1] or S-nitrosylated GSH (GSNO), resulted in a rapid and dose-dependent loss of enzymatic activity. Similarly, both isoforms were inactivated by $\mathrm{H}_{2} \mathrm{O}_{2}$, in a time- and dose-dependent manner. In order to characterize the interaction between free radicals and MAT, it was important to know whether this inactivation was reversible by reducing agents. When either NO- or $\mathrm{H}_{2} \mathrm{O}_{2}$-inactivated MAT were incubated with increasing concentrations of GSH, enzymatic activity was recovered. The ability of sulphydryl agents to reactivate MAT upon inhibition by free radicals, provide an insight about the nature of the interaction between MAT and these reactive species. In the first place, this interaction must be of covalent type. It probably involves the oxidation/nitrosylation of an enzyme site or sites, crucial for enzymatic activity. Second, the fact that MAT inactivation occurs at low concentrations of reactive species, and that it is readily reversible by reduced thiols, further supports the hypothesis of this enzyme being a finely tuned sensor and effector of redox signals.

Finally, we have addressed how specificity is achieved, one of the most urgent questions with respect to redox signaling. The complex chemistry and high reactivity of many of these species and their individual or simultaneous presence in the cell at a given time, may account for part of this specificity. Nevertheless, for this chemical signaling special attention must be paid to the target itself. 
With respect to MAT, we have studied whether the interaction of free radicals showed any functional specificity towards one or more of the ten cysteine residues present in the protein.

For this purpose all cysteine residues were replaced individually, by site-directed mutagenesis, for serine residues. The interaction of either $\mathrm{NO}$ or $\mathrm{H}_{2} \mathrm{O}_{2}$ with each of these mutants was tested in vitro, and in both cases we observed that the mutant lacking cysteine at position 121 was resistant to free radical-mediated inactivation. This residue is located at a "flexible loop" of the protein close to the active site, exposed to the surface and surrounded by a cationic environment which renders this thiol highly reactive and susceptible to oxidation. These features, together with the fact that the "flexible loop" in which cysteine 121 is located probably controls the access of substrates to the MAT active site [13], provide this enzyme with a specific molecular switch amenable to redox modulation.

\section{Physiological and pathological relevance of MAT interaction with free radicals}

MAT plays an essential function in liver metabolism. It is not solely implicated in the catabolism of methionine and the synthesis of AdoMet, but MAT activity also controls cellular GSH levels, polyamine synthesis and folate cycling [9]. From this, it can be inferred that chronic MAT inactivation is likely to contribute to the liver damage observed in several liver disorders, in which free radicals are produced in excess. However, this may be the result of the overstimulation of an otherwise

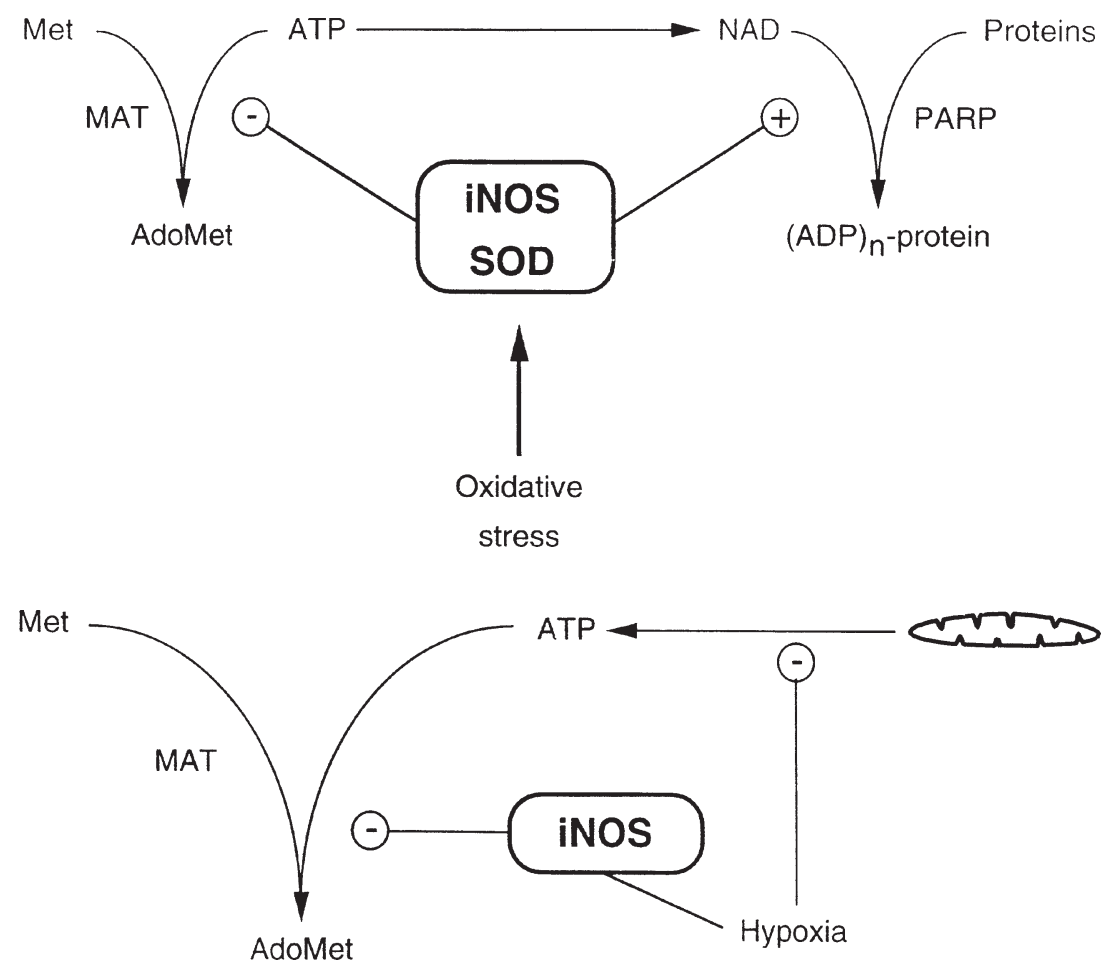

Fig. 1. A model for the control of hepatic consumption of ATP during oxidative stress and hypoxia: role of methionine adenosyltransferase. Top panel: under oxidative stress free radical generating enzymes are induced. The reactive species generated activate ATP-consuming enzymes such us poly ADP ribose polymerase (PARP). Bottom panel: under hypoxia ATP synthesis is decreased. In both situations MAT is inactivated by free radicals. MAT inactivation might result in a reduction in ATP consumption. This would contribute to the preseration of cellular viability. 
physiological regulatory mechanism. The reason for the existence of a redox switch on MAT may be found if we look at the other substrate of this enzyme, ATP. It is known that the administration of large quantities of methionine to rats results in the depletion of liver ATP [6]. Moreover, we have demonstrated that overexpression of rat liver MAT in CHO cells leads to a depletion of ATP and NAD levels [11], making these cells more sensitive to oxidative damage. Thus MAT activity may compromise, under certain conditions, cellular ATP levels and reductive capacity. MAT inactivation by free radicals could represent a transient adaptive response to an oxidative or nitrosative stress, aimed at the preservation of ATP levels (Fig. 1). On the other hand, we must note that interrupting MAT function for long periods of time would impair the many important cellular processes previously mentioned to depend on MAT. It is worth mentioning that MAT regulation by free radicals is restricted to the liver. Indeed, while liver MAT contains a redox sensitive cysteine residue at position 121, this amino acid is not present in the extrahepatic isoenzyme, an enzymatic form resistant to sulphydryl reagents [9]. This fact represents another level of specificity in redox signaling based also in the susceptibility of the target, which in this case depends on the isoenzyme expressed in a particular tissue. The exclusive regulation of liver MAT by free radicals, may originate from the need of a tight control of MAT activity in this organ. Indeed, MAT specific activity is highest in liver compared to other tissues; this organ metabolizes almost $50 \%$ of dietary methionine, always at the cost of cellular ATP. Thus ATP consumption could be diverted towards other pathways depending on cellular needs; this would be signaled by reactive oxygen and/or nitrogen species.

\section{Conclusions}

We have described the interaction of MAT with free radicals derived from oxygen and nitrogen. In this sense, MAT can be considered now as a new participant in the growing network of redox signaling. Liver MAT meets the requirements for being both a sensor and a transducer of the chemical signal carried by these reactive species into functional responses. We propose that the inactivation of MAT by these agents, could be a way to control hepatic consumption of ATP, consequently aimed at the preservation of cellular viability under different stress conditions.

\section{References}

[1] M.A. Avila, J.M. Mingorance, M.L. Martinez-Chantar, M. Casado, P. Martín-Sanz, L. Bosca and J.M. Mato, Regulation of rat liver S-adenosylmethionine synthetase during septic shock: role of nitric oxide, Hepatology 25 (1997), 391-396.

[2] T. Billiar, The delicate balance of nitric oxide and superoxide in liver pathology, Gastroenterology 108 (1995), 603605.

[3] H.F. Bunn and R.O. Poyton, Oxygen sensing and molecular adaptation to hypoxia, Physiol. Rev. 76 (1996), 839-885.

[4] C. Cabrero, A. Martin-Duce, S. Alemany and J.M. Mato, Specific loss of the high-molecular weight form of Sadenosyl-L-methionine synthetase in human liver cirrhosis, Hepatology 8 (1988), 1530-1534.

[5] F.J. Corrales, P. Ochoa, C. Rivas, M. Martín-Lomas, J.M. Mato and M.A. Pajares, Inhibition of glutathione synthesis in the liver leads to S-adenosyl-L-methionine synthetase reduction, Hepatology 14 (1991), 528-533.

[6] D.F. Hardwick, D.A. Applegarth, D.M. Cockcroft, P.M. Ross and R.J. Calder, Pathogenesis of methionine-induced toxicity, Metabolism 19 (1970), 381-391.

[7] L.J. Ignarro, Signal transduction mechanism involving nitric oxide, Biochem. Pharmacol. 41 (1991), 485-490.

[8] H.M. Lander, An essential role for free radicals and derived species in signal transduction, FASEB J. 11 (1997), $118-124$.

[9] J.M. Mato, L. Alvarez, P. Ortiz and M.A. Pajares, S-adenosylmethionine synthesis: molecular mechanisms and clinical implications, Pharmacol. Therap. 73 (1997), 265-280.

[10] C. Nathan, Nitric oxide as a secretory product of mammalian cells, FASEB J. 6 (1992), 3051-3064. 
[11] E. Sánchez-Góngora, J.G. Pastorino, L. Alvarez, M.A. Pajares, C. Garcia, J.R. Viña, J.M. Mato and J.L. Farber, Increased sensitivity to oxidative injury in Chinese hamster ovary cells stably transfected with rat liver S-adenosylmethionine synthetase cDNA, Biochem. J. 319 (1996), 767-773.

[12] M. Sundaresan, Z.-X. Yu, V.J. Ferrans, K. Irani and T. Finkel, Requirement for generation of $\mathrm{H}_{2} \mathrm{O}_{2}$ for platelet-derived growth factor signal transduction, Science 270 (1995), 296-299.

[13] F. Takusagawa, S. Kamitori and G.D. Markham, Structure and function of S-adenosylmethionine synthetase with ADP, BrADP, and $\mathrm{PP}_{2}$ at $2.8 \AA$ A resolution, Biochemistry 35 (1996), 2586-2596. 